\title{
3D Shape Measurement of Underwater Objects Using Motion Stereo
}

\author{
Hideo SAITO Hirofumi KAWAMURA Masato NAKAJIMA \\ Department of Electrical Engineering, Keio University \\ 3 - 14 - 1 Hiyoshi Kouhoku-ku Yokohama 223, Japan \\ phone: + 81-45-563-1141 (ext.3309), fax: + 81-45-563-2773, E-mail : saito@ozawa.elec.keio.ac.jp
}

\begin{abstract}
It is very difficult to measure 3D shape of underwater object from outside of the water by the use of conventional range image sensors, because the measured image is distorted by the effect of the light refraction on the water surface. In this study, we propose the new method for measuring 3D shape of underwater object by the use of motion stereo images obtained with a moving camera over the water surface. In this method, we employ an extended motion stereo method. We derive an equation of a locus of an object point in temporal-spatial space obtained by motion stereo images. The locus is parameterized by the position of the object point and the distance between the camera and the water surface. To obtain the parameters, all edge points in the images are voted into parameter space, like Hough transform. First, the parameter of the water surface distance is determined by the voting. Next, 3D position of the object point can be obtained by finding peak position in parameter space. We performed an experiment for demonstrating the efficacy of the proposed method. In the experiment, shape of an underwater polyhedron is measured by the proposed method. The experimental results show that the proposed method can provide not only $3 \mathrm{D}$ shape of underwater object but also the distance to the water surface.
\end{abstract}

\section{Introduction}

Many methods are proposed for reconstructing 3D shape by the use of images obtained with a CCD camera. Binocular stereo [1] method is the most popular technique to obtain 3D information of the scene. 3D information is provided by finding corresponding points between two images. Existing methods for finding the corresponding points are not perfect because they provide some incorrect correspondence. To reduce such mistaken corresponding points, motion stereo methods [2]-[4] are proposed. Since the motion stereo methods use image sequence obtained by moving camera, incorrect corresponding points can be decreased, and then the resultant 3D shape becomes more accurate.

However, these 3D shape measurement method can not be applied to the measurement of underwater scene because of the light refraction. Since the epipolar line can not be uniquely determined in the case for underwater scene, correspondence between stereo images can not be found in the conventional way. The epipolar line depends on the distance between camera and water surface. Therefore, the distance must be determined before finding the correspondence.

For solving this problem, we apply an extended motion stereo method in this study. The motion stereo scheme provides a temporal-spatial space from the image sequence taken with a moving camera. A feature point on the objective shape forms a locus in the temporalspatial space provided by motion stereo images. When any water effect does not exist, the locus becomes a line, which is often called as epipolar line. On the contrary, the locus in case of underwater point is not a line but a curve of which equation can be parameterized by the position of the point and the distance between camera and water surface. The distance is unique for every underwater point when the motion locus of the camera is parallel to the water surface. Under such condition, the distance between the camera and water surface can easily be determined because the distance is independent from the $3 \mathrm{D}$ shape to be measured. To determine the distance, we apply voting technique like a Hough transform [5]. After obtaining the distance, 3D position of the edge points in the motion stereo images are also determined by voting technique.

We also demonstrate the efficacy of the proposed method by experiments. In the experiments, we reconstruct 3D shape of underwater polyhedron by using motion stereo images obtained by a moving CCD camera. This summary also show the reconstructed shape of the polyhedron. 

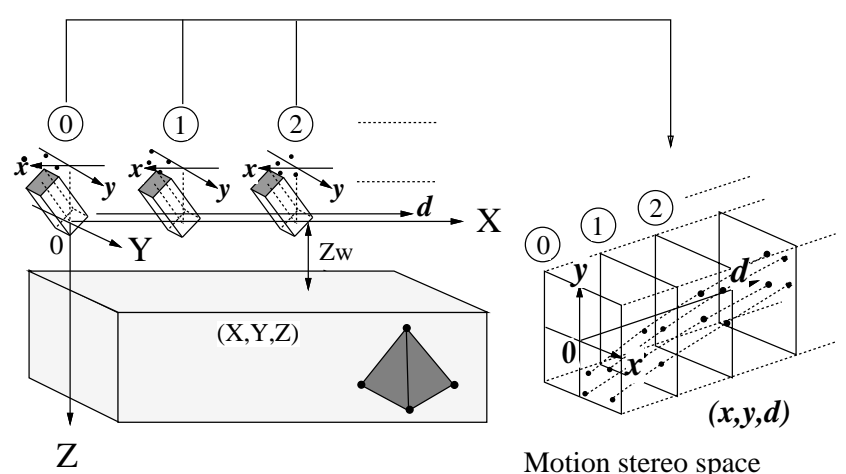

Motion stereo space

Figure 1: Basic arrangement for measuring 3D shape of underwater object.

\section{Principle of the Proposed Method}

Figure 1 shows the basic arrangement for measuring 3D shape of underwater object in this method.

A camera linearly moves along $X$ axis outside of water. The distance between the camera and the water surface is $Z_{w}$. Images are obtained at some positions of the camera on $X$ axis. The camera position is represented by $d$. Then the motion stereo space, $(x, y, d)$, can be considered by image sequence of frames obtained by the moving camera, where $x$ and $y$ show coordinate of camera image. Before forming the motion stereo space, obtained images are transformed onto the image place which is parallel to the water surface.

In such scheme, if there is no water above the object, feature points on the object form linear loci in the motion stereo space. Therefore, the conventional motion stereo technique [3] detects line, and calculates slope of the line which is corresponding to $3 \mathrm{D}$ position of the feature point. However, the loci are not linear for the case of underwater objects because of refraction on water surface. In this study, we derive an equation of the locus of underwater object for $3 \mathrm{D}$ shape measurement of underwater object.

In the motion stereo space $(x, y, d)$, the locus of a feature point on underwater object is expressed as

$$
\begin{gathered}
X-\frac{x}{A L} Z-\frac{x(A-1)}{A L} Z_{w}-d=0, \\
Y=(X-d) \frac{y}{x}
\end{gathered}
$$

where

$$
A=\sqrt{\left(N^{2}-1\right) \frac{x^{2}+y^{2}}{L^{2}}+N^{2}},
$$

and $L$ shows distance between the image plane and the focus point, and $N$ is the refraction index of water. If the

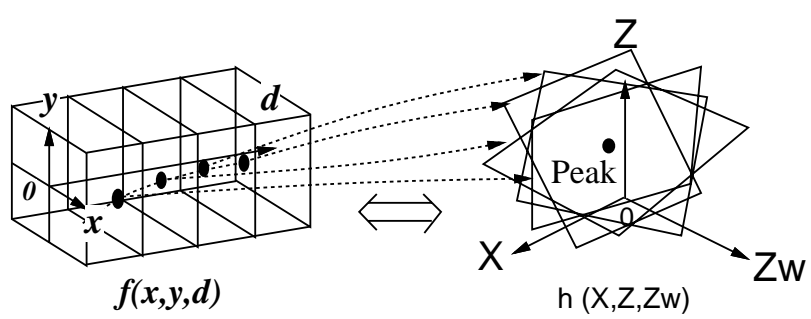

Motion stereo space

Parameter space

(a) In case of single feature point

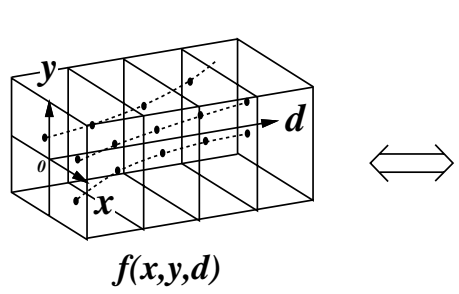

Motion stereo space

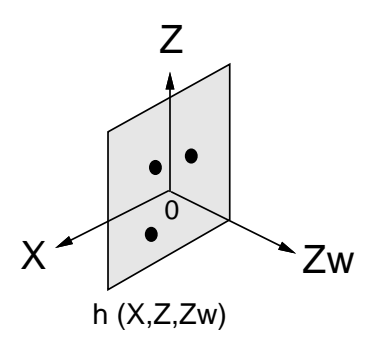

Parameter space (b) In case of multiple feature points

Figure 2: Relationship between motion stereo space and parameter space.

locus can be detected for a point in $X Y Z$ space, the $3 \mathrm{D}$ position of the point can be calculated from the locus.

Since the actual motion stereo images have many feature points, there are many loci in the motion stereo space. Therefore, each locus can not be found easily. In this method, a voting technique like a Hough transform [5] is applied to find each locus for each feature point in $X Y Z$ space. The locus equation (1) and (2) can be parameterized by $X, Z$, and $Z_{w}$. These parameters provide the $3 \mathrm{D}$ position of corresponding feature point.

Considering parameter space of $\left(X, Z, Z_{w}\right)$, a point in the motion stereo space $(x, y, d)$ is corresponding to a planar locus expressed as equation (1). figure 2(a). The loci of every point corresponding to the same feature point in $X Y Z$ space intersect each other at a point in the parameter space as shown in figure 2(a). Then the parameters $\left(X, Z, Z_{w}\right)$ of the intersection point are corresponding to the position of the feature point $(X, Z)$ and the distance to the water surface $Z_{w}$. If there are some feature points in $X Y Z$ space, there are also some loci in the motion stereo space, and then there are some peaks in the parameter space as shown in figure $2(b)$.

If each point in the motion stereo space is voted onto corresponding planar locus in the parameter space,the peaks of the voted number provide actual parameter sets of $\left(X, Z, Z_{w}\right)$. Consequently, if peaks of the voted number 


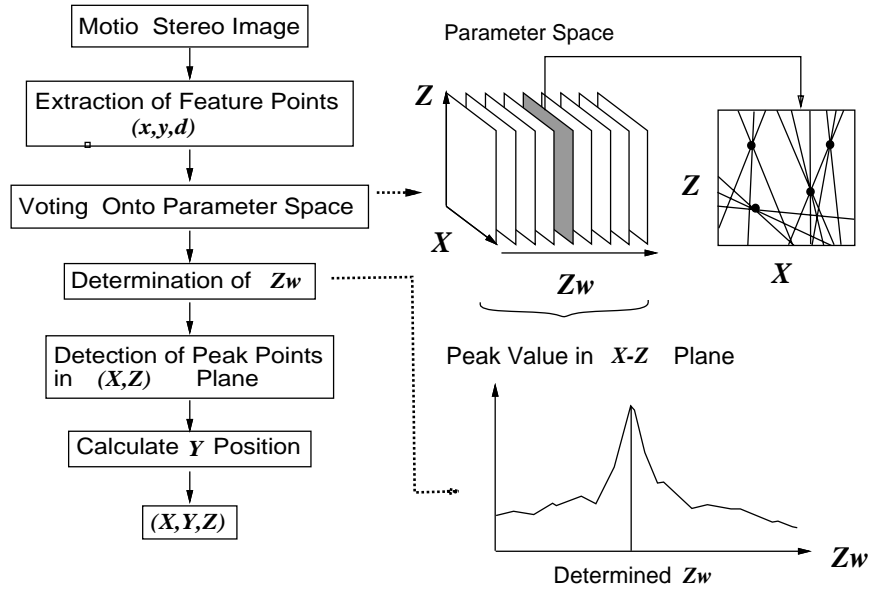

Figure 3: Flow of algorithm for detecting peak parameter.

in the parameter space can be extracted, the position of each peak provides a locus parameter corresponding to an object point in $X Y Z$ space.

Figure 3 shows flow of the algorithm for detecting the peak parameter. While the peak position on $X-Z$ place depends on shape of measured object, the peak position on $Z_{w}$ axis is constant because $Z_{w}$ is the distance between the camera and the water surface. Therefore, for detecting the peak position in the parameter space, peak position on $Z_{w}$ direction is first found and then $Z_{w}$ is first determined. Next, peak position on $X-Z$ plane corresponding to the peak $Z_{w}$ are found for obtaining 3D position of the feature point. In this way, a peak parameter is detected. Finally, 3D position of the feature point in $X Y Z$ space can be determined from detected $X, Z$, and $Z_{w}$.

\section{3D Shape Measurement of Underwater Polyhedron}

As shown in the previous section, some feature points must be detected from image. In this sense, polyhedron is suitable for the proposed method because the edge points of the polyhedron can be easily found as feature points. In this section, the algorithm for measuring shape of underwater polyhedron is described.

Figure 4 shows the flow of the measuring algorithm. For each image in the image sequence obtained by the moving camera, points of vertices as feature points are detected by the conventional edge detection operator. Every feature point $(x, y, d)$ is voted onto the corresponding locus in the parameter space. As described in the previous section, the distance between the camera and the

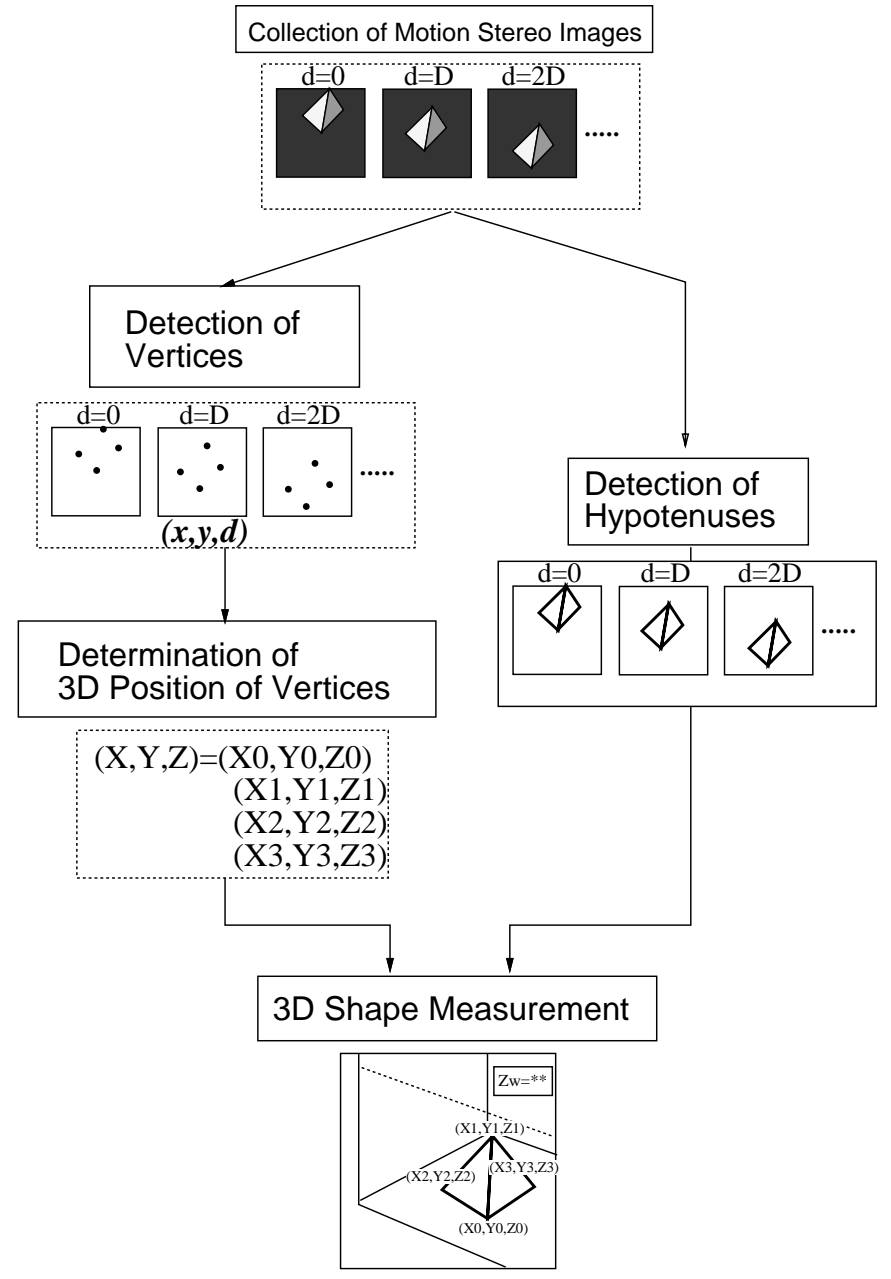

Figure 4: Flow for measuring shape of underwater polyhedron.

water surface $Z_{w}$ is first determined, and then $X$ and $Y$ and $Z$ of all edge point are obtained by finding the peak position of the voted number in the parameter space $\left(X, Z, Z_{w}\right)$.

Edge lines of the polyhedron are also detected from the motion stereo images to connect measured 3D points of the vertices. Then, 3D position of the edge lines are also provided by interpolating the $3 \mathrm{D}$ position of the vertices. Finally, 3D shape of the polyhedron can be reconstructed.

\section{Experiment}

We performed an experiment to measure 3D shape of a underwater polyhedron by the use of an experimental arrangement as shown in figure 5 . The conditions of the experimental setup are shown in figure 6. Each motion 
Figure 5: Experimental arrangement for measuring 3D shape of underwater object.

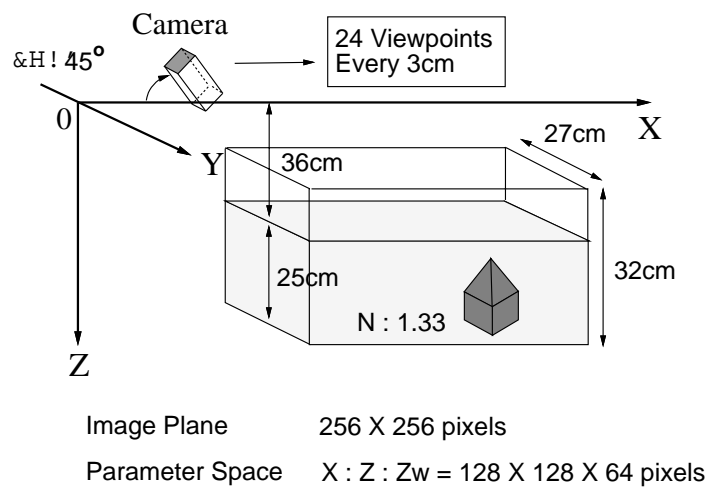

Figure 6: Conditions of the experimental setup.

stereo image is obtained at every $3 \mathrm{~cm}$.

Figure 7 shows an example of the motion stereo images. Figure 7 (a) shows motion images of underwater object. For comparison, figure 7 (b) shows motion stereo images obtained at the corresponding camera position in case of no water. Because of the refraction at the water surface, both images are different although they are obtained at the same camera position.

Figure 8 shows the measured shape of the polyhedron. which is obtained from the motion stereo images as shown in figure 7 (a), by the proposed method. The line image of measurement shape is synthesized at the same viewpoint as the motion stereo images of $d=30.0 \mathrm{~cm}$ shown in figure 7 . The measurement result image is closer to the motion stereo image without water (figure $7(\mathrm{~b})$ ) than that for the underwater object (figure $7(\mathrm{a})$ ). This shows that the proposed method effectively works for correcting the refraction effect in the motion stereo image.
Table 1: Measured length of the edge lines of the polyhedron is presented. The actual length of all the edge lines is $10.0 \mathrm{~cm}$. Edge (1)-(2) represents the edge between vertices (1) and (2), for example. The measured position of the vertices are : (1) (93.0,4.0,43.0)! "(2)(92.0,-1.0,55.0)!" (3) $(89.0,9.0,57.0)$ ! " $(4)(98.0,12.0,56.0)$ ! "

(5) $(92.0,-1.0,64.0)$ ! "(6)(89.0,9.0,66.0).

(a) Measured length using measured $Z_{w}$.

\begin{tabular}{|c|c||c|c|}
\hline Edge & Length $[\mathrm{cm}]$ & Edge & Length $[\mathrm{cm}]$ \\
\hline \hline$(1)-(2)$ & 13.0 & $(2)-(5)$ & 11.0 \\
\hline$(1)-(3)$ & 15.4 & $(3)-(4)$ & 9.5 \\
\hline$(1)-(4)$ & 16.1 & $(3)-(6)$ & 9.0 \\
\hline$(2)-(3)$ & 10.7 & $(5)-(6)$ & 10.3 \\
\hline
\end{tabular}

(b) Measured length using correct $Z_{w}$.

\begin{tabular}{|c|c||c|c|}
\hline Edge & Length $[\mathrm{cm}]$ & Edge & Length $[\mathrm{cm}]$ \\
\hline \hline$(1)-(2)$ & 10.7 & $(2)-(5)$ & 7.3 \\
\hline$(1)-(3)$ & 12.6 & $(3)-(4)$ & 9.9 \\
\hline$(1)-(4)$ & 11.6 & $(3)-(6)$ & 7.1 \\
\hline$(2)-(3)$ & 10.9 & $(5)-(6)$ & 9.7 \\
\hline
\end{tabular}

To show the measurement error of the $3 \mathrm{D}$ positions of vertices of the polyhedron, we compare the measured length of edge lines with the actual length. The actual length of all the edge lines is $10.0 \mathrm{~cm}$. The measured length by the proposed method is shown in table 1 (a). This table shows that some errors are included in the measured 3D shape. In the proposed method, the distance between camera and water surface $Z_{w}$ is first determined before the $3 \mathrm{D}$ positions $(X, Y, Z)$ of feature points. Therefore, estimation error of $Z_{w}$ is induced in the error of $3 \mathrm{D}$ position estimation. In the previous experiment, the error of $Z_{w}$ was $4.0 \mathrm{~cm}$. The estimated $Z_{w}$ was $32.0 \mathrm{~cm}$ while the actual one was $36.0 \mathrm{~cm}$. If $Z_{w}$ can be determined more accurately, the accuracy in measuring $3 \mathrm{D}$ position will be improved. In table 1 (b), the measured length of the edge in case of correct $Z_{w}$ is shown. The length in table 1 (b) is more accurate than that shown in table 1 (a). Accordingly, the measurement accuracy of $Z_{w}$ is important for obtaining accurate shape reconstruction by the proposed method.

\section{Conclusion}

We proposed a new method for measuring 3D shape of underwater object by the use of motion stereo technique. The efficacy of the proposed method can be demonstrated by the experiment for measuring 3D shape of underwater polyhedron. 
(a) Motion stereo images in case of underwater object.

(b) Motion stereo images without water.

Figure 7: Examples of motion stereo images.

In the presented experiment, the object is limited to polyhedrons, so that the feature points can be detected easily. In case of more complicate object, it will be more difficult to detect the peak of feature points in the parameter space, because there will be a lot of feature points in the motion stereo images. Therefore, the algorithm for the peak detection must be improved in the future work.

\section{References}

[1] B.K.P.Horn, Robot Vision, pp.299-333, MIT Press, 1986.

[2] G.Xu, S.Tsuji, and M.Asada, "A motion stereo method based on coarse-to-fine control strategy", IEEE Trans. Patt. Anal. Mach. Intel., vol.PAMI-9, pp.332-336, 1987.

[3] M. Yamamoto, "Determinating three-dimensional structure from image sequences given by horizontal and vertical moving camera", Trans. IEICE, vol.J69D, pp.1631-1639, 1986. (in Japanese)

[4] Y.T.Zhou and R.Chellapa, Artificial Neural Networks for Computer Vision, pp.44-62, SpringerVerlag, 1992.

[5] D.H.Ballard and C.M.Brown, Computer Vision, pp.123-131, Prentice Hall, 1982.

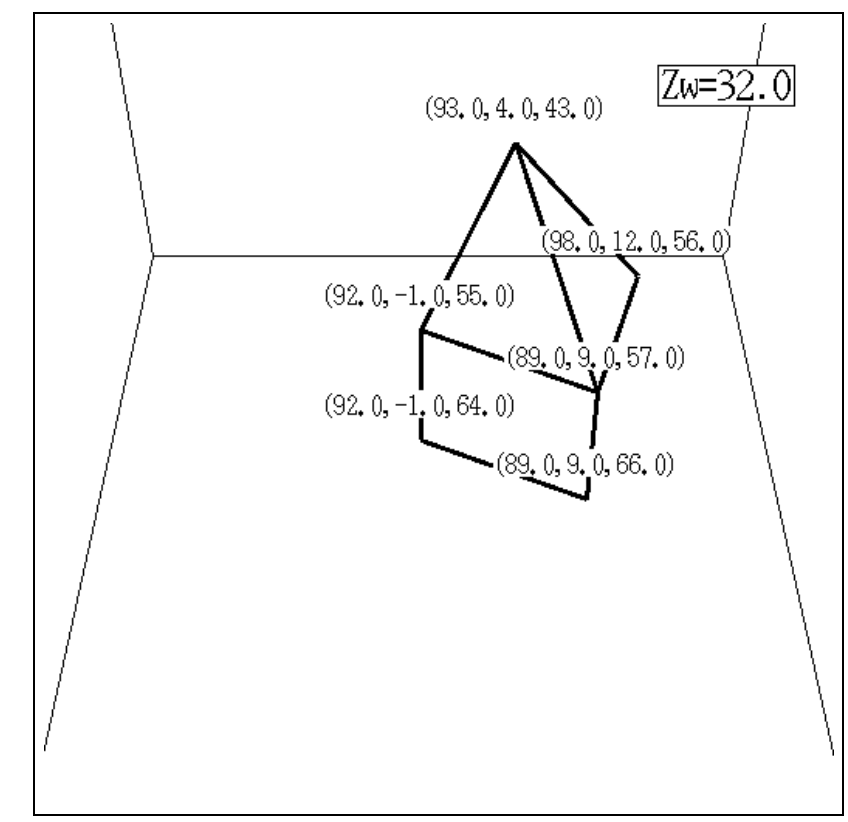

Figure 8: Reconstructed shape of the underwater polyhedron (unit:cm). 